\title{
Temperature dependence of magnetization-induced second harmonic generation at buried exchange-biased interfaces
}

\author{
V. K. Valev, ${ }^{1, *}$ M. Gruyters, ${ }^{2}$ A. Kirilyuk, ${ }^{1}$ and Th. Rasing ${ }^{1}$ \\ ${ }^{1}$ Institute for Molecules and Materials, Radboud University Nijmegen, Toernooiveld 1, 6525ED, Nijmegen, the Netherlands \\ ${ }^{2}$ Humboldt-Universität zu Berlin, Institut für Physik, Newtonstrasse 15, D-12489 Berlin, Germany
}

(Received 5 May 2006; published 2 June 2006)

\begin{abstract}
We have investigated the temperature dependence of magnetization-induced optical second harmonic generation $(\mathrm{MSHG})$ in the exchange-biased $\mathrm{CoO} / \mathrm{Cu} / \mathrm{Fe}$ multilayer. Below the blocking temperature, there is a strong MSHG response from the $\mathrm{CoO} / \mathrm{Cu}$ interface which, for large exchange bias values, equals and even dominates the contribution of the ferromagnetic Fe interfaces. In a previous publication we showed that there is a correlation between exchange bias and the MSHG asymmetry. Here we demonstrate the relationship between the second harmonic Kerr rotation and exchange bias and, based on that, we offer additional evidence that pinned uncompensated spins are present beyond the range of $\mathrm{Cu}$ spacer thickness where a hysteresis loop shift is observed.
\end{abstract}

DOI: 10.1103/PhysRevB.73.233101

PACS number(s): 75.70.Cn, 42.65.Ky, 75.30.Gw, 75.30.Et

Exchange bias has been discovered almost 50 years ago, ${ }^{1}$ yet despite the intense research efforts on the subject, ${ }^{2,3}$ there is still no complete theoretical explanation for the phenomenon. Recently, its exploitation in exchange-biased devices, such as hard disk drives and magnetic random access memory ${ }^{4}$ has renewed research interest. The effect is obtained when a ferromagnet (FM)/antiferromagnet (AFM) bilayer is cooled from above the Néel temperature but below the Curie temperature, in the presence of an external magnetic field. Its main characteristics are: a shift $H_{\mathrm{E}}$ of the hysteresis loop away from zero field and, generally, an increase of the coercivity $H_{\mathrm{C}}$. The temperature below which the effect occurs is called the blocking temperature $T_{\mathrm{B}}$.

Recent research suggests that a small number of pinned uncompensated AFM spins at the interface might be the origin of the loop shift., ${ }^{5,6}$ Other recent studies revealed a longrange nature of exchange bias: upon insertion of a nonmagnetic spacer layer between the FM and the AFM, the authors observed a decrease of the effect with increasing spacer thickness as measured with the hysteresis loop shift. ${ }^{7-10}$ This suggests that the number of pinned uncompensated AFM spins diminishes and becomes zero with increasing spacer thickness, though a clear interpretation of this observation is still lacking. The study of these spins and their behavior at buried interfaces with different experimental approaches is therefore of prime importance for the understanding of their role in the exchange bias process. In particular, for direct observation it is desirable that the experimental responses from the relevant interface can be distinguished from those of other magnetic interfaces.

Magnetization-induced second harmonic generation (MSHG) has been known to exhibit an excellent sensitivity to interfacial magnetism ${ }^{11}$ and has been previously employed to the study of exchange bias on a different type of multilayers (without spacer) although the observed effects were very subtle. ${ }^{12}$ In an earlier communication, ${ }^{13}$ we have demonstrated how MSHG can be applied to the study of the interfacial spins responsible for exchange bias in a $\mathrm{CoO} / \mathrm{Cu} / \mathrm{Fe}$ system. Furthermore, we found evidence that the exchange bias interaction propagates even further across the $\mathrm{Cu}$ spacer than the range estimated from the loop shift measurements.

In this paper, we have applied the interface-sensitive technique of MSHG to investigate the temperature dependence of exchange bias in the $\mathrm{CoO} / \mathrm{Cu} / \mathrm{Fe}$ system after positive and negative field cooling. We observe that for large exchange bias values, the nonlinear magneto-optical susceptibility of the sample is dominated by the tensor elements associated with the $\mathrm{CoO} / \mathrm{Cu}$ interface rather than by those associated with the ferromagnetic Fe interfaces. This indicates that MSHG offers the possibility of a direct observation of the spins responsible for exchange bias. Furthermore, in an independent experimental approach that provides an alternative way of investigating the pinned uncompensated AFM spins with MSHG, we demonstrate the relationship between the second harmonic Kerr rotation and exchange bias. Based on that, we offer additional supportive evidence that pinned uncompensated spins are present beyond the range of $\mathrm{Cu}$ spacer thickness where a hysteresis loop shift is observed. Moreover, our results offer deeper insight into the MSHG asymmetry sensitivity to the pinned uncompensated spins.

The basic structure of our layered samples was $\mathrm{Si}(111) / \mathrm{Fe} / \mathrm{Cu} / \mathrm{CoO} / \mathrm{Au}$. Initially, $6 \mathrm{~nm} \mathrm{Fe}$ was deposited by molecular beam epitaxy (MBE) on hydrogen-passivated $\mathrm{Si}(111),{ }^{14}$ followed by a $\mathrm{Cu}$ layer of $2.5 \mathrm{~nm}$ thickness. Different $\mathrm{Cu}$ thicknesses were deposited for the experiment in Fig. 3. After the preparation of $2 \mathrm{~nm} \mathrm{CoO},{ }^{15}$ the sample was covered by a $6 \mathrm{~nm}$ Au cap layer to prevent contamination from the atmosphere. The Fe film possesses a single crystalline bcc(110) surface orientation ${ }^{16}$ while the $\mathrm{CoO}$ consists of densely packed roundly shaped particles. Because the $\mathrm{CoO}$ does not reveal any x-ray diffraction peaks, it is assumed to be amorphous.

Exchange bias was induced by cooling the sample from a temperature of $300 \mathrm{~K}$, in the presence of an external magnetic field of $2.5 \mathrm{kOe}$. Hysteresis loops extended from -4.5 to $+4.5 \mathrm{kOe}$.

MSHG measurements were carefully performed ${ }^{17}$ using a Ti:sapphire laser at $800 \mathrm{~nm}$ wavelength with a pulse width of $\sim 100 \mathrm{fs}$ and a repetition rate of $82 \mathrm{MHz}$. The laser power 


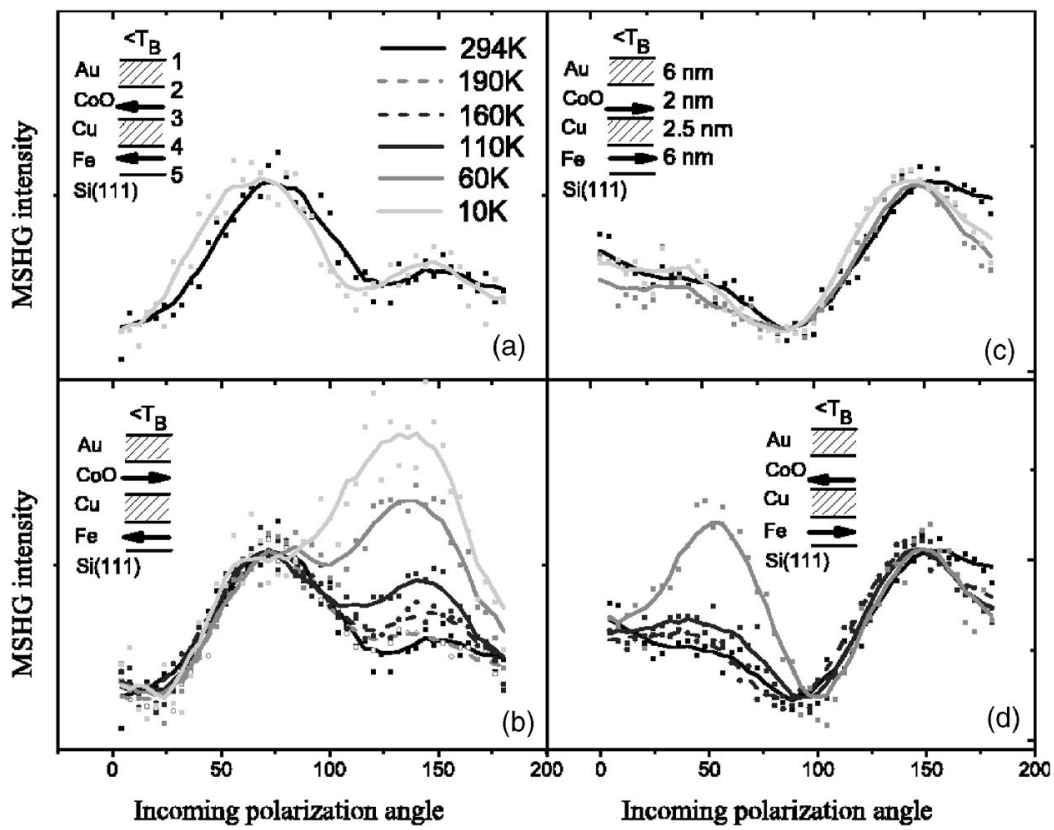

FIG. 1. MSHG intensity as function of the rotation of the fundamental light polarization from the $P_{\text {in }}$ direction at different temperatures, after negative field cooling (a) and after positive field cooling (b) for $\mathbf{M}_{\mathrm{Fe}}<0$ and after positive (c) and negative (d) field cooling for $\mathbf{M}_{\mathrm{Fe}}>0$. The analyzer was fixed along the $S_{\text {out }}$ direction. The signal was normalized to the room temperature response. In the inset, the magnetization at the sample interfaces above and below $T_{\mathrm{B}}$ is represented, indicating the parallel [(a) and (c)] and antiparallel $[(b)$ and (d)] configurations. was $5 \mathrm{~mW}$ and the light was focused to a spot with a diameter of $\sim 100 \mu \mathrm{m}$. The angle of incidence was $30^{\circ}$ and the magnetic field was applied in the longitudinal configuration, i.e., in the direction where the plane of the sample surface intersects the plane of optical incidence (for further details see Ref. 11). In all experiments, the light polarization was linear.

For intense electromagnetic fields $\mathbf{E}(\omega)$, such as those generated by a pulsed laser beam incident on a thin multilayer film, the polarization at the harmonic frequency $2 \omega$ is given by

$$
\mathbf{P}_{i}^{(l)}(2 \omega)=\chi_{i j k}^{(l)} \mathbf{E}_{j}^{l}(\omega) \mathbf{E}_{k}^{l}(\omega),
$$

where $\chi_{i j k}$ is a third order polar tensor describing the second order nonlinear optical (NLO) susceptibility at the symmetry breaking interface between the centrosymmetric films and $l$ numbers the interfaces in our sample. ${ }^{13}$ We can separate two types of contributions to the susceptibility: the "magnetic" $\left(\chi_{m}\right)$ and "nonmagnetic" $\left(\chi_{n m}\right)$, depending on whether the tensor elements associated with them change sign upon reversal of the magnetic moment. Note that the non-magnetic part also includes the NLO response from defects or microstructure effects, that will not reverse sign after field cooling in an opposite external magnetic field.

The nonzero net magnetic moment at the $\mathrm{CoO}$ interfaces is related to the exchange bias and its sign can be reversed if the sample is field cooled in an opposite magnetic field. We can distinguish two types of configurations: parallel $(P)$ and antiparallel (AP), which are related to the relative orientation of the FM Fe layer and the pinned uncompensated spins at the $\mathrm{CoO} / \mathrm{Cu}$ interface, see insets in Fig. 1. In the case of the AP configuration, the sample was field cooled down to the temperature of measurement and then the orientation of the FM layer was reversed by reversing the external magnetic field.

For a fixed polarizer-analyzer combination, the second- order susceptibility $\chi$ at any given interface can be described by a single number. ${ }^{18,19}$ In the limit of ultrathin films we can combine those susceptibilities (the interfaces are numbered as in the inset in Fig. 1)

$$
\begin{gathered}
\chi_{m}^{P}=\chi_{m}^{(3)}+\left(\chi_{m}^{(4)}+\chi_{m}^{(5)}\right), \\
\chi_{m}^{A P}=\chi_{m}^{(3)}-\left(\chi_{m}^{(4)}+\chi_{m}^{(5)}\right), \\
\chi_{n m}=\chi_{n m}^{(1)}+\chi_{n m}^{(2)}+\chi_{n m}^{(3)}+\chi_{n m}^{(4)}+\chi_{n m}^{(5)} .
\end{gathered}
$$

Note that here we have excluded a possible contribution of the $\mathrm{Au} / \mathrm{CoO}$ interface $\chi_{m}^{(2)}$ (as shown previously ${ }^{13}$ ). A potential contribution from the AFM ordering in CoO (Ref. 20) is incorporated in the nonmagnetic part as it should be symmetrical with respect to the direction of the field cooling.

The second harmonic intensity for the parallel and antiparallel configurations is then given by

$$
I^{P / A P}=\left|\chi_{n m} \pm \chi_{m}\right|^{2} I^{2},
$$

where $I$ is the intensity of the incoming fundamental light. For observing an MSHG contrast, only the sign change of $\chi_{m}$ relative to $\chi_{n m}$ is relevant. In nonabsorbing materials, the relative phase between these susceptibilities is $90^{\circ}$ and therefore they do not interfere. However, in the case of our samples interference is allowed and the relative phase can be considered to be temperature independent as the optical properties of the sample do not change noticeably in the temperature range that we consider. ${ }^{21}$

So far, it has been shown that reversing the magnetization in ferromagnetic materials changes the sign of $\chi_{m} \cdot{ }^{11}$ Here, we demonstrate that such a change can also be obtained when reversing the direction of the spins responsible for exchange bias at the antiferromagnetic/spacer interface.

Figure 1 shows the MSHG intensity as function of the rotation of the fundamental light polarization at different temperatures. During the experiments in Figs. 1(a) and 1(b), 


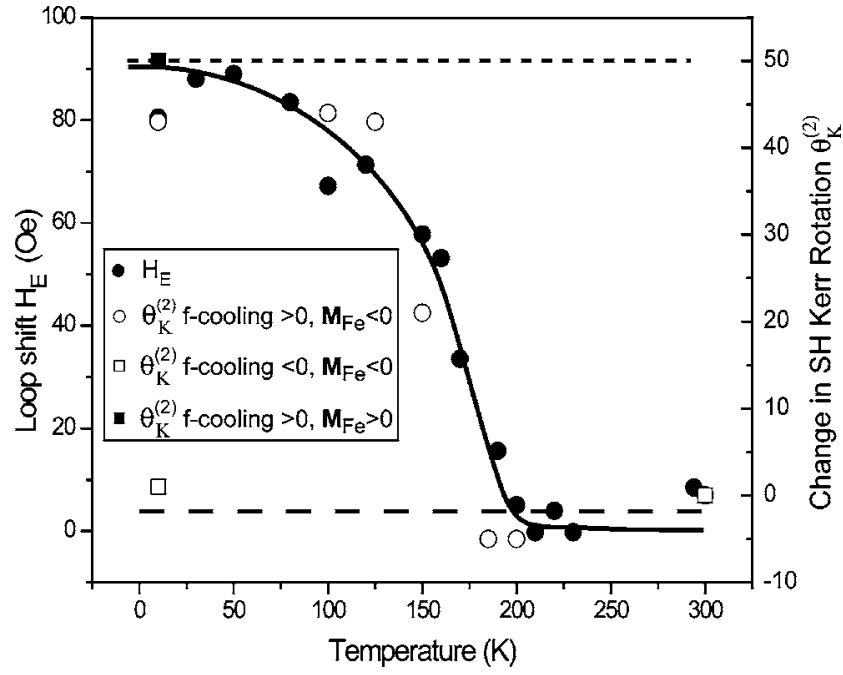

FIG. 2. The exchange bias loop shift $H_{\mathrm{E}}$ and the change in the second harmonic Kerr rotation as function of temperature. $\mathbf{H}_{\mathrm{EX}}$ and $\mathbf{M}_{\mathrm{Fe}}<0$, after positive (empty circles) and negative (empty squares) field cooling. The polarizer was fixed along the $S_{\text {in }}$ direction while $-10^{\circ}$ corresponds to the $P_{\text {out }}$ direction of the analyzer.

the applied external field $\left(\mathbf{H}_{\mathrm{EX}}\right)$ and therefore the magnetization at the $\mathrm{Fe}$ interfaces $\left(\mathbf{M}_{\mathrm{Fe}}\right)$ was along the negative direction (i.e., $\mathbf{H}_{\mathrm{EX}}, \mathbf{M}_{\mathrm{Fe}}<0$ ). In Fig. 1(a), the MSHG signal in the parallel configuration can be seen above $T_{\mathrm{B}}\left(T_{\mathrm{B}}\right.$ $\sim 185 \mathrm{~K}$, see Fig. 2) and after field cooling to $10 \mathrm{~K}$. The curves exhibit two peaks: a large one at $\sim 70^{\circ}$ and a smaller one at $\sim 145^{\circ}$. We observe that the two curves are very similar. On the other hand, Fig. 1(b) shows the MSHG response in the antiparallel configuration above $T_{\mathrm{B}}$ and after field cooling. Again, we see that above $T_{\mathrm{B}}(T=294 \mathrm{~K}$ and $T$ $=190 \mathrm{~K}$ ), the curves are similar, however below $T_{\mathrm{B}}$ the signal starts changing with decreasing temperature, indicating a polarization rotation due to the appearance of new tensor components- $\chi_{m}^{(3)}$. The latter are related to the appearance of a magnetic symmetry at the interface, due to the pinned uncompensated spins at the $\mathrm{CoO} / \mathrm{Cu}$ interface.

The nature of this change can be better understood when we look at Fig. 1(c). Here, we plotted again the MSHG intensity as function of the rotation of the fundamental polarization at different temperatures, but this time $\mathbf{H}_{\mathrm{EX}}, \mathbf{M}_{\mathrm{Fe}}$ $>0$. We can see that above $T_{\mathrm{B}}$, the curves exhibit two peaks close to the angles in Fig. 1(a) but having an inverse relationship: there is a large one at $\sim 145^{\circ}$ and a smaller one at $\sim 60^{\circ}$. Therefore, the temperature dependence of the SHG polarization in Fig. 1(b) corresponds to a transition from the magnetization state of Fig. 1(a) to the magnetization state in Fig. 1(c). In other words, we can conclude that the change in the MSHG response in Fig. 1(b) at $T<T_{\mathrm{B}}$ is due to a reversal of the sign of the total magnetic part of the NLO susceptibility in the sample. The same can be seen in Fig. 1(d). Thus, the MSHG technique allows us not only to detect $\chi_{m}^{(3)}$, which originates from the pinned uncompensated spins at the $\mathrm{CoO} / \mathrm{Cu}$ interface, but also shows that its absolute value becomes larger than those from $\chi_{m}^{(4)}$ and $\chi_{m}^{(5)}$, which are associated with the $\mathrm{Fe}$ interfaces.

Another way of examining the $\chi_{m}^{(3)}$ tensor components is

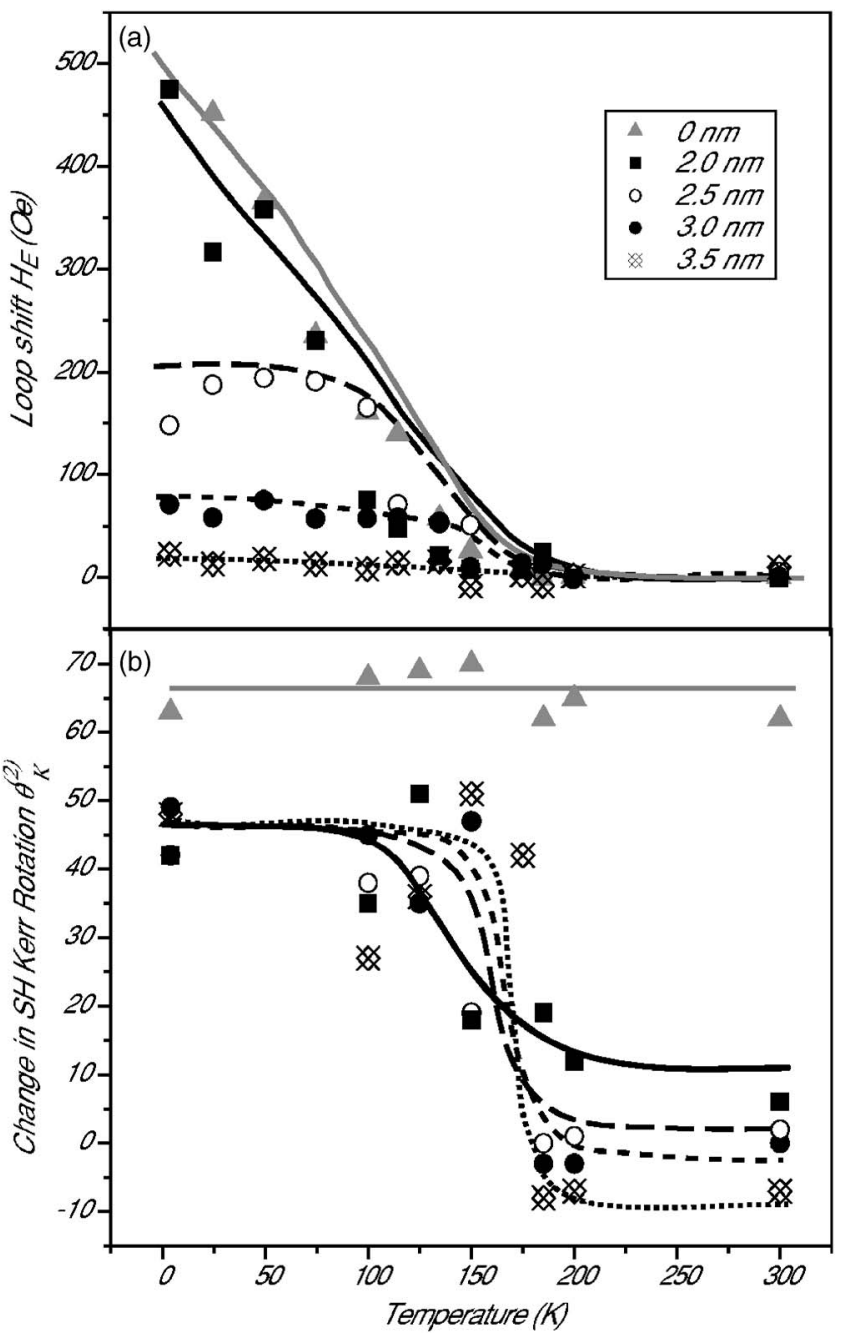

FIG. 3. The exchange bias loop shift $H_{\mathrm{E}}$ (a) and the second harmonic Kerr rotation (b) as function of temperature for different $\mathrm{Cu}$ spacer thicknesses. The lines are guides to the eye. The polarizer was fixed along the $S_{\text {in }}$ direction while $-10^{\circ}$ corresponds to the $P_{\text {out }}$ direction of the analyzer.

by studying the nonlinear optical Kerr rotation $\theta_{K}^{(2)}$. This is done by performing the following experiment: the fundamental polarization was fixed while the analyzer was rotated. Fig. 2 shows the thus obtained MSHG Kerr rotation angle at different temperatures (during the measurements, $\mathbf{H}_{\mathrm{EX}}, \mathbf{M}_{\mathrm{Fe}}$ $<0$ ). We can see that for positive field cooling (antiparallel configuration) there is a clear change in $\theta_{K}^{(2)}$ from $0^{\circ}$ to $50^{\circ}$, which exactly follows the variations of the exchange bias loop shift $H_{\mathrm{E}}$. On the other hand, for negative field cooling (parallel configuration) there is no change in $\theta_{K}^{(2)}$ with temperature.

Note that $50^{\circ}$ is the direction of the MSHG polarization for $\mathbf{H}_{\mathrm{EX}}, \mathbf{M}_{\mathrm{Fe}}>0$ after positive field cooling. This is a parallel configuration, and since we have established that for parallel configurations there is no or little change occurring with temperature, we can conclude that again the change of the second harmonic Kerr rotation angle from $0^{\circ}$ to $50^{\circ}$ must be attributed to a sign change in $\chi_{m}$ !

This sign reversal can be explained in the following way. 
Above $T_{\mathrm{B}}$, the sign of $\chi_{m}$ is solely determined by $\chi_{m}^{(4)}$ and $\chi_{m}^{(5)}$. When the sample is field cooled in the direction in which the measurement is performed, (i.e., parallel configuration), the appearance of $\chi_{m}^{(3)}$ does not affect this sign [see Eq. (2a)]. There is only a change in the absolute value of $\chi_{m}^{P}$ and it does not have a very pronounced influence on the MSHG signal since the magnetization of the sample is saturated. However, when the sample is field cooled in one direction after which the external field is reversed, [i.e., antiparallel configuration, see Eq. (2b)], the sign of $\chi_{m}$ changes for $\left|\chi_{m}^{(3)}\right|>\left|\chi_{m}^{(4)}+\chi_{m}^{(5)}\right|$ and, hence, the large MSHG response that is observed. The observation that we see an effect in the antiparallel configuration and not in the parallel one may also indicate that MSHG is particularly sensitive to $\chi_{m}^{(3)}$ through the frustrations between the AFM pinned uncompensated spins and the FM ones.

The consequences of this finding are quite general. The fact that the signal generated by $\chi_{m}^{(3)}$, i.e., originating from the pinned uncompensated spins at the $\mathrm{CoO} / \mathrm{Cu}$ interface, dominates the contribution from the $\mathrm{Fe}$ interfaces suggests that MSHG can be used for direct observation of the exchange bias related spins in a large variety of samples. Indeed, depending on the fundamental wavelength, the angle of incidence and the refraction indices of the materials, it is in principle possible to optically "quench" the contributions to the MSHG signal from the FM interfaces while at the same time enhancing the response from the relevant AFM interface.

Understanding that there is a clear difference in the MSHG response between the parallel and antiparallel configurations gives us additional insight into the sensitivity of the MSHG asymmetry $A=\left(I^{P}-I^{A P}\right) /\left(I^{P}+I^{A P}\right)$ to the temperature variations of exchange bias that we have found previously. Clearly, it is the variations of $I^{A P}$ that give rise to this sensitivity, i.e., the changes of the value of $\chi_{m}$, including its sign reversal.

To investigate the MSHG sensitivity to the pinned uncompensated spins at the $\mathrm{CoO} / \mathrm{Cu}$ interface as a function of the $\mathrm{Cu}$ spacer thickness, Fig. 3 shows the observed loop shift $H_{\mathrm{E}}$ and $\theta_{K}^{(2)}$ as function of temperature for various $\mathrm{Cu}$ spacers. We can see that for a $\mathrm{Cu}$ thickness of $3.5 \mathrm{~nm}$, the loop shift in Fig. 3(a) is zero, while the second harmonic Kerr rotation in Fig. 3(b) still indicates the presence of pinned uncompensated spins. This result is consistent with our earlier experiment that monitored the MSHG asymmetry $(A)$ derived from hysteresis loop measurements (Ref. 11, Fig. 2). It is important to notice that the data in Fig. 3 were obtained from a different experiment. They provide additional evidence for the presence of pinned uncompensated spins at the $\mathrm{CoO} / \mathrm{Cu}$ interface for a $\mathrm{Cu}$ thickness of $3.5 \mathrm{~nm}$. Although the conditions for the two measurements are different, both are sensitive to $\chi_{m}^{(3)}$.

In conclusion, we have demonstrated that for rotating incoming and outgoing polarization, in the antiparallel configuration there is a strong MSHG signal below $T_{\mathrm{B}}$, associated with the $\mathrm{CoO} / \mathrm{Cu}$ interface. The observed large second harmonic Kerr rotation $\theta_{K}^{(2)}$ is shown to be proportional to the exchange bias loop shift $H_{\mathrm{E}}$. For large exchange bias values (below $60 \mathrm{~K}$ ) the MSHG signal equals and even dominates the contribution of the ferromagnetic Fe interfaces to the nonlinear magneto-optical susceptibility of the sample. Based on the second harmonic Kerr rotation dependence on magnetic order at the $\mathrm{CoO} / \mathrm{Cu}$ interface, we offer additional evidence that pinned uncompensated spins at this interface remain present even though the hysteresis loop shift indicates no exchange bias effect.

This work was supported in part by the EU project DYNAMICS, the Dutch organization for Fundamental Research on Matter (FOM), and the Dutch NanoNed program.
*Electronic address: v.valev@science.ru.nl

${ }^{1}$ W. H. Meiklejohn and C. P. Bean, Phys. Rev. 102, 1413 (1956).

${ }^{2}$ J. Nogués and I. K. Schuller, J. Magn. Magn. Mater. 192, 203 (1998).

${ }^{3}$ A. E. Berkowitz and K. Takano, J. Magn. Magn. Mater. 200, 552 (1999).

${ }^{4}$ J. C. S. Kools, IEEE Trans. Magn. 32, 3165 (1996).

${ }^{5}$ H. Ohldag, A. Scholl, F. Nolting, E. Arenholz, S. Maat, A. T. Young, M. Carey, and J. Stöhr, Phys. Rev. Lett. 91, 017203 (2003).

${ }^{6}$ P. Kappenberger, S. Martin, Y. Pellmont, H. J. Hug, J. B. Kortright, O. Hellwig, and E. E. Fullerton, Phys. Rev. Lett. 91, 267202 (2003).

${ }^{7}$ N. J. Gökemeijer, T. Ambrose, and C. L. Chien, Phys. Rev. Lett. 79, 4270 (1997).

${ }^{8}$ M. Gruyters, M. Gierlings, and D. Riegel, Phys. Rev. B 64, 132401 (2001).

${ }^{9}$ T. Mewes, B. F. P. Roos, S. O. Demokritov, and B. Hillebrands, J. Appl. Phys. 87, 5064 (2000).

${ }^{10}$ M.-T. Lin, C. H. Ho, C.-R. Chang, and Y. D. Yao, Phys. Rev. B
63, 100404(R) (2001).

${ }^{11}$ A. Kirilyuk and Th. Rasing, J. Opt. Soc. Am. B 22, 148 (2005).

${ }^{12}$ L. C. Sampaio, A. Mougin, J. Ferre, P. Georges, A. Brun, H. Bernas, S. Poppe, T. Mewes, J. Fassbender, and G. Hillebrands, Europhys. Lett. 63, 819 (2003).

${ }^{13}$ V. K. Valev, M. Gruyters, A. Kirilyuk, and Th. Rasing, Phys. Rev. Lett. 96, 067206 (2006).

${ }^{14}$ G. S. Higashi, Y. J. Chabal, G. W. Trucks, and K. Raghavachari, Appl. Phys. Lett. 56, 656 (1990).

${ }^{15}$ M. Gruyters and D. Riegel, Phys. Rev. B 63, 052401 (2001).

${ }^{16}$ M. Gruyters, Surf. Sci. 515, 53 (2002).

${ }^{17}$ V. K. Valev, M. Gruyters, A. Kirilyuk, and Th. Rasing, Phys. Status Solidi B 242, 3027 (2005).

${ }^{18}$ J. E. Sipe, J. Opt. Soc. Am. B 4, 481 (1987).

${ }^{19}$ Y. Z. Wu, R. Vollmer, H. Regensburger, X.-F. Jin, and J. Kirschner, Phys. Rev. B 63, 054401 (2001).

${ }^{20}$ M. Fiebig, Th. Lottermoser, V. V. Pavlov, and R. V. Pisarev, J. Appl. Phys. 93, 6900 (2003).

${ }^{21}$ V. V. Pavlov, R. V. Pisarev, A. Kirilyuk, and Th. Rasing, Phys. Rev. Lett. 78, 2004 (1997). 